University of Nebraska - Lincoln

DigitalCommons@University of Nebraska - Lincoln

\title{
Cost Efficiency of Anycast-Based Forwarding in Duty-Cycled WSNs with Lossy Channel
}

\author{
Yuyan Xue \\ University of Nebraska-Lincoln, yxue@cse.unl.edu \\ Mehmet C. Vuran \\ University of Nebraska-Lincoln, mcvuran@cse.unl.edu \\ Byrav Ramamurthy \\ University of Nebraska-Lincoln, bramamurthy2@unl.edu
}

Follow this and additional works at: https://digitalcommons.unl.edu/cseconfwork

Part of the Computer Sciences Commons

Xue, Yuyan; Vuran, Mehmet C.; and Ramamurthy, Byrav, "Cost Efficiency of Anycast-Based Forwarding in Duty-Cycled WSNs with Lossy Channel" (2010). CSE Conference and Workshop Papers. 153.

https://digitalcommons.unl.edu/cseconfwork/153

This Article is brought to you for free and open access by the Computer Science and Engineering, Department of at DigitalCommons@University of Nebraska - Lincoln. It has been accepted for inclusion in CSE Conference and Workshop Papers by an authorized administrator of DigitalCommons@University of Nebraska - Lincoln. 


\title{
Cost Efficiency of Anycast-Based Forwarding in Duty-Cycled WSNs with Lossy Channel
}

\author{
Yuyan Xue, Mehmet C. Vuran and Byrav Ramamurthy \\ Department of Computer Science and Engineering \\ University of Nebraska-Lincoln \\ Lincoln, NE 68588-0115 USA \\ \{yxue, mcvuran,byrav\}@cse.unl.edu
}

\begin{abstract}
Anycasting has been proposed recently as an efficient communication method for asynchronous duty-cycled wireless sensor networks. However, the interdependencies between end-toend communication cost and the anycasting design parameters have not been systematically studied. In this paper, a statistical endto-end cost model is presented to capture the end-to-end latency and energy consumption of anycasting operation under a realistic wireless channel model. By exploring the relationship between the end-to-end cost efficiency and the forwarding decision dependent anycasting design parameters, two anycasting forwarding metrics are proposed for fully distributed forwarding decision. By exploring the relationship among the preamble length, the size of the forwarding set and the achievable end-to-end cost efficiency, a series of preamble length control guidelines are proposed for low and extremely low duty-cycled WSNs. According to our analytical results and simulation validation, the proposed forwarding metrics help reduce the end-toend latency and energy consumption by about $55 \%$ for anycasting with moderate preamble length, compared with the existing heuristic forwarding metrics. The proposed preamble length control guidelines help reduce, by more than half, the end-to-end energy and latency costs in low and extremely-low duty-cycled WSNs.
\end{abstract}

\section{INTRODUCTION}

Anycast-based forwarding (anycasting for short) has been proposed as an efficient communication technique for asynchronous duty-cycled Wireless Sensor Networks (WSNs) [1]. Compared with the traditional unicast-based forwarding schemes, where each sensor node has a single next-hop node specified by the routing metric, that needs to be awakened before the data packet transmission, anycasting technique does not specify a fixed next-hop node at the sender. Instead, the sender utilizes the broadcast nature of preamble/RTS packet transmission to wake up neighboring nodes and forms a forwarding set. All awake nodes within the forwarding set are then prioritized according to a specific forwarding metric. The node with the highest priority becomes the next-hop node to respond with a CTS packet and receive the data packet.

The performance of an anycasting design mainly depends on two important design factors: (1) Prioritization Policy: How the neighboring nodes of a sender should be prioritized so that the most suitable awake node in the forwarding set can be selected as the next-hop to reduce the end-to-end communication cost. (2) Wake-up Policy: How much time and energy should be spent in the neighboring node wake-up process so that the most suitable node can be selected from a reasonably sized forwarding set to forward the packet and reduce the overall communication cost? To investigate how these design factors determine the anycasting performance and drive cost efficient anycasting design, we provide a statistical end-to-end cost model for basic anycasting operation under a realistic wireless channel model. Based on the model, we use the cumulative distribution function of two end-to-end metrics: latency-coefficient and energy-coefficient, to characterize the cost-efficiency of an anycasting design with specific design parameters. By quantitatively analyze the relationship between the achievable end-to-end cost efficiency and the neighboring prioritization/wake-up policy, we propose a series of packet forwarding and preamble length control guidelines for anycasting design to significantly reduce the end-to-end energy and latency cost in duty-cycled WSNs.

The rest of the paper is organized as follows. Section II describes the existing anycasting analysis frameworks and points out their limitations. Section III describes the assumptions and the network model considered in this paper. Section IV describes an end-to-end anycasting cost-model for single flow transmission under a realistic log-normal channel model. Sections V and VI discuss how to design cost-efficient neighboring node prioritization and wake-up policies,respectively, based on our proposed cost model. The analytical results derived in Sections V and VI are validated through the simulation results presented in Section VII. The paper is concluded in Section VIII.

\section{RELATED WORK}

GeRaF [2] is one of the earliest anycasting designs to improve the end-to-end communication throughput in WSNs. A series of studies [1], [3], [4], [5], [6] have been conducted later to model and optimize the design parameters of GeRaFtype anycasting, sometime classified as opportunistic routing, designs. These studies focus on a simple anycasting model, where the neighboring node prioritization policy only depends on the node geographic location. The simple anycasting model tries to minimize the end-to-end hop count through global optimized or distributed greedy forwarding metrics. Based on the ideal disc channel model, end-to-end hop count minimization guarantees optimal end-to-end performance because possible retransmissions are not considered for the data package forwarding between the sender and selected forwarder. However, wireless links in practical WSN settings can be extremely 


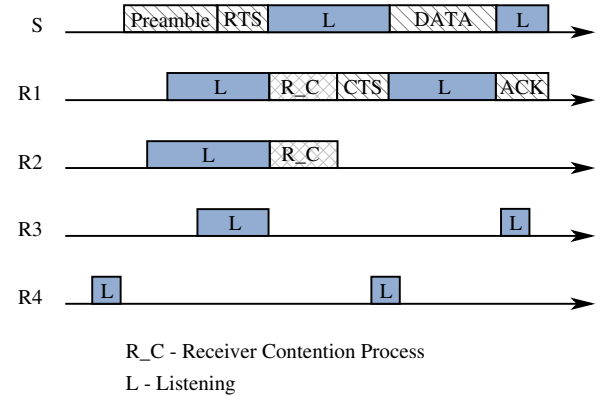

Fig. 1. Timeline of RTS-CTS and DATA-ACK packet exchange between a sender, $\mathrm{S}$, and its neighboring nodes, R1-R4, in anycasting.

unreliable, deviating to a large extent from the ideal channel models used in these works.

The work proposed in [7] provides an anycasting performance analysis under lossy channel and suggests using new channel-quality-aware forwarding metrics in neighboring node prioritization policy. However, how the duty-cycle operation and wake-up policy affect the end-to-end communication cost is not considered in the analysis framework. Traditionally, the unicast forwarding sender, such as in BMAC [8], uses a long preamble to wake up the neighboring nodes so that the packet can always be forwarded to the most suitable node among all of its neighbors for communication cost minimization. In contrast, for most existing anycasting schemes, the sender uses a short RTS packet to wake up its neighbors and form a nonempty forwarding set, in which, however, the most suitable node among all neighboring nodes may not be included. As a result, the packet may end up being forwarded to a suboptimal next-hop node, which however, costs less for forming a smaller forwarding set. Clearly, a systematic analysis on how such a tradeoff affects the end-to-end cost-efficiency under realistic channel model is vital for efficient anycast design, which however, has not been addressed in the literature.

\section{AsSUMPTIONS AND System Model}

\section{A. Assumptions}

We consider a WSN composed of sensor nodes with location awareness but not global topology awareness. The nodes are randomly deployed with a uniform distribution. Duty-cycle operation is adopted in the network so that each sensor node switches between sleep and awake states periodically. The sensing data are collected by the sensor nodes and converge-casted to the sink. This study focuses on lowrate/time-scheduled applications such as habitat monitoring, where interference is at a minimum (or nonexistent) [7]. Interference is an important factor to consider, specially in medium and heavy traffic scenarios, and is a subject of our future work.

\section{B. Basic Anycasting Operation}

The basic anycasting operation consists of two continuous handshake processes, as we discuss below and show in Fig. 1.

Control Packet Handshake: The sender ( $S$ in Fig. 1) sends a preamble followed by an RTS packet to wake up the neighboring nodes, meanwhile setting up a timer. If the timer expires before a CTS packet is received, the sender retransmits

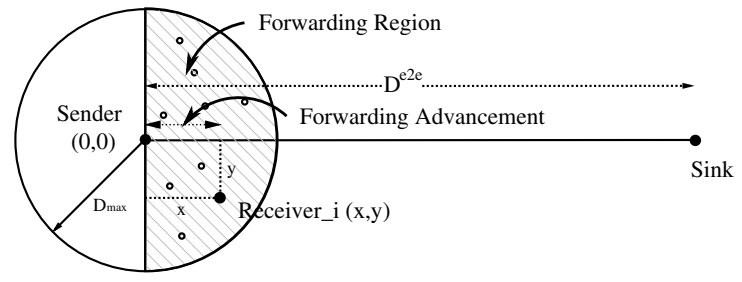

Fig. 2. Coordination illustration for single-hop anycasting operation.

the RTS packet and resets the timer. Any neighboring node that overhears the preamble frame stays awake, and becomes an Active Receiver (AR). The ARs that successfully receive the RTS packet and are located within the forwarding region of the sender, as shown in Fig. 2, become Potential Receivers (PR). The rest of the ARs switch to sleep mode immediately and follow the default duty-cycle from then on (R3 in Fig.1). All the potential receivers then start the receiver contention process, where each potential receiver prioritizes its potential as the next hop according to a specific forwarding metric. By using a polling contention period based channel contention strategy proposed in [9], a PR that has the highest priority can obtain the channel within a guaranteed number of time slots and respond to the sender with a CTS packet containing its node ID (R1 in Fig. 1). We call this node as the Winning Receiver (WR). Other potential receivers will switch back to the sleep period and follow the default duty-cycle design (R2 in Fig. 1).

Data Packet Handshake: After a successful control packet handshake between the sender and the winning receiver, the data packet forwarding is accomplished by the data packet handshake. In a data packet handshake, the sender will unicast the DATA packet to the winning receiver, whose node ID is indicated in the CTS packet. The sender also sets up a timer for the data packet handshake process. Upon receiving the DATA packet, the winning receiver responds to the sender with an ACK packet. If the timer expires before an ACK packet is received, the sender retransmits the DATA packet and resets the timer.

Note that, some variations of anycasting design, such as those proposed in [10], [11], consider forwarding without Preamble and RTS/CTS exchange. Any neighboring node that receives the DATA packet competes for being the most suitable forwarder. Since the length of the DATA packet is usually much longer than the RTS/CTS packet, the packet error rate is usually high for data packet handshake. In duty-cycled WSNs, the size of forwarding set could become very small or close to zero in these anycasting variations, which in turn results in large number of retransmissions. Therefore, different analytical models and design policies need to be developed for these variations based on different forwarding operations, which are out of the scope of this paper.

\section{Channel Model}

The log-normal channel model proposed in [12] is used to capture the signal attenuation and dynamics of the wireless channel. We consider the DSSS-OQPSK (Offset Quadrature Phase Shift Keying with Direct Sequence Spread 
Spectrum) as the modulation scheme, which is adopted by the commonly used CC2420 transceiver in MicaZ and TelosB motes [13]. The expected PRR achieved at distance $D$, for a packet with length $l$ bits can be found as $\Psi(D, l)=\left(1-Q\left(\sqrt{\frac{2 B}{R} \cdot 10^{\frac{\Gamma_{\sigma}(D)}{10}}}\right)\right)^{l}$, where $\Gamma_{\sigma}(D)=$ $P_{t}-P_{L}\left(D_{0}\right)-10 \eta \log _{10}\left(\frac{D}{D_{0}}\right)-P_{n}+X_{\sigma} \cdot Q(D)=$ $\frac{1}{\sqrt{2 \pi}} \int_{D}^{\infty} e^{-\frac{t^{2}}{2}} d t$. $R$ is the radio communication bit rate. $B$ is the noise bandwidth; $P_{t}$ is the transmit power in $\mathrm{dBm} . P_{L}\left(D_{0}\right)$ is the path loss at a reference distance $D_{0}$ in $\mathrm{dBm}$. $\eta$ is the path loss exponent. $D$ is the distance between the wireless sender and receiver. $P_{n}$ is the receiver-end noise power. $X_{\sigma} \sim \mathcal{N}(0, \sigma)$ is the shadow fading component represented as a Normal-distribution random variable with mean 0 and variance $\sigma$. The expectation of $\Psi(D, l)$ is thus given as

$$
\bar{\Psi}(D, l)=\frac{1}{2 \pi \sigma} \int_{-\infty}^{+\infty} \Psi(D, l) * e^{\frac{-(\gamma-\mu(D))^{2}}{2 \sigma^{2}}} d \gamma,
$$

where $\mu(D)=P_{t}-P_{L}\left(D_{0}\right)-10 \eta \log _{10}\left(\frac{D}{D_{0}}\right)-P_{n}$. Note that $\Psi(D, l)$ is a random variable. Therefore, no cut-off value exists as the maximum transmission range for a sender. Instead, a valid definition for disconnection distance, $D_{\max }$, under lognormal channel model is given, at which the wireless links have a high probability $\left(>p_{H}\right)$ of having low packet reception rates $\left(<\Psi_{L}\right)$ [12]. By defining proper threshold values $p_{H}=$ 0.96 , and $\Psi_{L}=0.1$ [7], the disconnection distance can be derived as $D_{\max }=10^{\frac{\gamma_{L}-\sigma Q^{-1}\left(1-p_{H}\right)-P_{t}+P_{n}+P_{L}\left(D_{0}\right)}{-10 \eta}}$, where $\gamma_{\ell}$ is the signal-to-noise ratio in $\mathrm{dBm}$ corresponding to $\Psi_{L}$. Based on above definition, we also define the network node density in terms of node degree $\rho$, where $\rho$ is the expected number of nodes within the area of disconnection distance, $D_{\max }$.

\section{Asynchronous Duty-Cycle Operation and Forwarding Set}

Asynchronous duty-cycle operation is adopted by each sensor node with a $t_{L}$ awake-period followed by a $t_{S}$ sleepperiod. The entire period of a duty-cycle is defined as $t_{D}$, where $t_{D}=t_{L}+t_{S}$. The duty-cycle is then derived as $d_{c}=\frac{t_{L}}{t_{D}}$. To ensure the recognition of a preamble during the listening period, the receiver must start its listening period before the last preamble slot is transmitted. The minimum intersection of listening period and preamble period is denoted as $t_{C}$. Based on the duty-cycle operation, the probability that a neighboring node is awakened by a preamble of length $t_{P}$ is given as,

$$
P_{\text {wake }}= \begin{cases}\frac{\left(t_{P}+t_{D} d_{c}-t_{C}\right)}{t_{D}} & t_{C} \leq t_{P} \leq t_{M}, \\ 1 & t_{P}>t_{M}\end{cases}
$$

where $t_{M}=t_{C}+t_{D}\left(1-d_{c}\right)$. From (2), we observe that when $t_{P}>t_{M}$, all the neighboring nodes within the forwarding region are guaranteed to be awakened by the preamble. Since $P_{\text {wake }}$ can be varied only when $t_{C} \leq t_{P} \leq t_{M}$, we define the normalized preamble length as $t_{P, n}=\left(t_{P}-t_{C}\right) /\left(t_{M}-\right.$ $\left.t_{C}\right)$. Based on (2), we derive some important values related to the size of forwarding set of anycasting operation. First, $\rho_{I}$ is defined as the expected number of neighboring nodes awakened by the preamble, i.e. the expected number of ARs, which is given as

$$
\rho_{I}=\rho \cdot P_{\text {wake }}
$$

Then $\rho_{I}^{R}$ is defined as the expected number of PRs, i.e., the expected number of nodes that participate in the receiver contention process. The PRs form the forwarding set, from which a winning receiver is selected by the prioritization policy specified by a forwarding metric. To find $\rho_{I}^{R}$, we first consider the probability that a neighbor node, that is randomly located within the disconnection distance, successfully receives the RTS packet, which is given as

$P_{R 0}=\frac{1}{2 \pi \sigma} \int_{-\infty}^{\infty} \int_{0}^{D_{\max }} \Psi\left(D, l_{R T S}\right) p(D) e^{\frac{-(\gamma-\mu(D))^{2}}{2 \sigma^{2}}} d D d \gamma$,

where $p(D)=2 D / D_{\max }^{2}$ is the probability of the node located at distance $D$ from the sender, and $l_{R T S}$ is the length of RTS packet in bits. Then $\rho_{I}^{R}$ is given as

$$
\rho_{I}^{R}=\rho^{\prime} \cdot P_{\text {wake }} \cdot P_{R 0},
$$

where $\rho^{\prime}=\frac{\rho}{2}$ is the expected number of neighbor nodes located within the forwarding region.

\section{END-TO-END COST MODEL}

In this section, we first provide a statistical analysis of transmission latency and energy consumption for single-hop anycasting operation. Based on that, we define the costcoefficient as the end-to-end metric to determine the latency efficiency and energy efficiency of an anycasting design with specific protocol parameters. The proposed end-to-end cost model is then used to facilitate the cost-efficient anycasting prioritization policy and wake-up policy design in duty-cycled WSNs.

\section{A. Single-Hop Transmission Latency}

First, we derive the expected transmission latency for a successful single-hop anycasting. For the control packet handshake, an RTS retransmission is issued whenever the sender is timed out for receiving the CTS packet. The expected time for a successful control packet handshake, $E\left[\mathcal{T}_{h / s}^{c t r l}\right]$, is then given by the required number of RTS packet retransmissions for a successful handshake and the time consumed for each handshake attempt. Let $T^{c t r l}=T_{P \mid R}+T_{R_{-} C}+T_{C}$, where $T_{P \mid R}, T_{C}$, and $T_{R_{-} C}$ are the time consumed for transmitting the (preamble+RTS) packet, for receiver contention, and for transmitting the CTS packet, respectively. $T_{t / o}^{c t r l}$ is the timeout value at the sender for RTS packet retransmission. Then

$$
E\left[\mathcal{T}_{h / s}^{c t r l}\right]=\left(\frac{1}{P_{R} \cdot P_{C}}-1\right) \cdot\left(T_{P \mid R}+T_{t / o}^{c t r l}\right)+T^{c t r l}
$$

where $P_{R}$ is the probability that the RTS packet is successfully received by at least one potential receiver, and $P_{C}$ is the probability that the CTS packet is successfully received by the sender. With the protocol-specific RTS and CTS packet lengths, $P_{R}$ and $P_{C}$ achieved by a potential receiver are calculated according to (1). If we assume $T_{t / o}^{c t r l}=T_{R_{-} C}+T_{C}$, i.e., the sender retransmits the (preamble+RTS) frame if no CTS is received after $T_{R_{-} C}+T_{C}$ time period, $E\left[\mathcal{T}_{h / s}^{\text {ctrl }}\right]$ is then simplified as 


$$
E\left[\mathcal{T}_{h / s}^{c t r l}\right]=T^{c t r l} /\left(P_{R} \cdot P_{C}\right) .
$$

The expected time for a successful data packet handshake is calculated similar to the control packet handshake as

$$
E\left[\mathcal{T}_{h / s}^{\text {data }}\right]=\left(\frac{1}{P_{D} \cdot P_{A}}-1\right) \cdot\left(T_{D}+T_{t / o}^{\text {data }}\right)+\left(T_{D}+T_{A}\right)
$$

where $P_{D}$ is the probability of the DATA packet being successfully received by the winning receiver, and $P_{A}$ is the probability of the ACK packet being successfully received by the sender. With the protocol-specific DATA and ACK packet lengths, $P_{D}$ and $P_{A}$ achieved by a potential receiver can also be calculated according to (1). $T_{D}$ is the time for transmitting the DATA packet, $T_{A}$ is the time for transmitting the ACK packet, and $T_{t / o}^{\text {data }}$ is the timeout value at the sender for DATA packet retransmission. Similarly, if we assume $T_{t / o}^{\text {data }}=T_{D}+T_{A}$,

$$
E\left[\mathcal{T}_{h / s}^{\text {data }}\right]=T^{\text {data }} /\left(P_{D} \cdot P_{A}\right),
$$

where $T^{\text {data }}=T_{D}+T_{A}$. According to (6) and (7), the expected time for a successful single-hop forwarding is

$$
E\left[\mathcal{T}^{\text {hop }}\right]=E\left[\mathcal{T}_{h / s}^{\text {ctrl }}\right]+E\left[\mathcal{T}_{h / s}^{\text {data }}\right]=\frac{T^{c t r l}}{P_{R} \cdot P_{C}}+\frac{T^{\text {data }}}{P_{D} \cdot P_{A}} .
$$

\section{B. Single-Hop Energy Consumption}

In this subsection, we focus on energy consumption analysis for a successful single-hop anycasting operation. The energy consumption at a node is derived using the product of its awake time, $t_{w}$, and the unit-time energy (i.e. power) consumption parameter $\varepsilon$. The energy consumption is not distinguished between transmitting and receiving states of a node while it is awake, because the unit-time energy consumption parameters, $\varepsilon$, in these two states are almost the same for popular transceivers $\left(\varepsilon \doteq 25 \mathrm{~mJ} / \mathrm{s}\right.$ when $\left.P_{t}=0 \mathrm{dBm}\right)$ used on most sensor nodes [13], [14]. The energy consumption at the sender and the receivers are derived separately. In case of an anycasting sender, it remains awake during the entire forwarding process. According to (8), the energy consumed at the sender for a successful single-hop forwarding is given as

$$
E\left[\mathcal{E}_{s}^{h o p}\right]=E\left[\mathcal{T}^{h o p}\right] \cdot \varepsilon
$$

In case of the anycasting receivers, we first consider the control packet handshake process, where different amount of energy is consumed by the active receivers, the potential receivers and the winning receivers. Let $E^{c t r l}=E_{P \mid R}+E_{R_{-} C}+E_{C}$, where $E_{P \mid R}=\rho_{I} \cdot\left[\frac{1}{2} \cdot T_{P \mid R} \cdot \varepsilon\right]$, according to (3), is the total amount of energy consumed by the active receivers for receiving the preamble followed by the RTS packet; $E_{R_{-} C}=$ $\rho_{I}^{R} \cdot\left[T_{R_{-} C} \cdot \varepsilon\right]$, according to (5), is the energy consumed by the potential receivers during the receiver contention process; $E_{C}=T_{C} \cdot \varepsilon$ is the energy consumed by the winning receiver in transmitting the CTS packet; the energy consumed at the receivers for a successful control packet handshake is then given as

$$
E\left[\mathcal{E}_{h / s, r}^{c t r l}\right]=\frac{E_{P \mid R}+P_{R}\left(E_{R_{-} C}+E_{C}\right)}{\left(P_{R} P_{C}\right)^{2}} .
$$

For the data packet handshake, since the winning receiver is the only awake neighboring node and remains awake during the handshake, the energy consumption at the receivers for a successful data packet handshake can be found as

$$
E\left[\mathcal{E}_{h / s, r}^{\text {data }}\right]=E\left[\mathcal{T}_{h / s, r}^{\text {data }}\right] \cdot \varepsilon=\frac{T^{\text {data }}}{P_{D} \cdot P_{A}} \cdot \varepsilon .
$$

Accordingly, the expected energy consumption at the receivers for a successful single-hop forwarding is given as

$$
\begin{aligned}
E\left[\mathcal{E}_{r}^{\text {hop }}\right] & =E\left[\mathcal{E}_{h / s, r}^{c t r l}\right]+E\left[\mathcal{E}_{h / s, r}^{\text {data }}\right] \\
& =\left[\frac{\frac{1}{2} \rho_{I} T_{P \mid R}+P_{R}\left(\rho_{I}^{R} T_{R_{-} C}+T_{C}\right)}{\left(P_{R} P_{C}\right)^{2}}+\frac{T^{\text {data }}}{P_{D} P_{A}}\right] \varepsilon
\end{aligned}
$$

Finally, according to (9) and (10), the expected energy consumption in the network for a successful single-hop forwarding is

$$
E\left[\mathcal{E}^{h o p}\right]=E\left[\mathcal{E}_{s}^{h o p}\right]+E\left[\mathcal{E}_{r}^{h o p}\right]
$$

\section{End-to-end Latency and Energy Cost}

In multi-hop WSNs, the end-to-end transmission cost depends not only on single-hop transmission cost but also on the number of hops required end-to-end. Therefore, the expected communication cost as a result of a single flow from a source node at distance $D^{e 2 e}$ from the sink, $E\left[C^{e 2 e}\right]$, is given as

$$
E\left[C^{e 2 e}\right]=E\left[C^{h o p}\right] \cdot E\left[H^{e 2 e}\right]=D^{e 2 e} \cdot \frac{E\left[C^{h o p}\right]}{E\left[A^{h o p}\right]},
$$

where $E\left[H^{e 2 e}\right]$ is the expected end-to-end hop count; $E\left[C^{h o p}\right]$ is the expected per-hop communication cost, such as perhop transmission latency or energy consumption; and $E\left[A^{h o p}\right]$ is the expected per-hop forwarding advancement, which is defined as the end-to-end distance decrement achieved in single-hop forwarding towards the sink, as shown in Fig. 2. For a single flow with fixed end-to-end distance, an efficient anycasting design aims at minimizing the component, $\frac{E\left[C^{h o p}\right]}{E\left[A^{h o p}\right]}$, i.e., the per-hop communication cost normalized by the expected per-hop forwarding advancement.

In order to capture the cost-efficiency of anycasting, based on (12), we define the end-to-end cost coefficient, $\mathcal{C}$, as

$$
\mathcal{C}=E\left[C^{h o p}\right] / E\left[A^{h o p}\right],
$$

which is independent of the end-to-end transmission distance. By substituting $E\left[C^{h o p}\right]$ with (8) and (11) separately, the endto-end latency coefficient, $\mathcal{C}^{T}$, is found as

$$
\mathcal{C}^{T}=\frac{1}{E\left[A^{\text {hop }}\right]} \cdot\left(\frac{T^{\text {ctrl }}}{P_{R} \cdot P_{C}}+\frac{T^{\text {data }}}{P_{D} \cdot P_{A}}\right)
$$

and the end-to-end energy coefficient, $\mathcal{C}^{E}$, is found as

$$
\mathcal{C}^{E}=\frac{\varepsilon\left[\frac{T^{c t r l}}{P_{R} P_{C}}+\frac{2 T^{\text {data }}}{P_{D} P_{A}}+\frac{\frac{1}{2} \rho_{I} T_{P \mid R}+P_{R}\left(\rho_{I}^{R} T_{R_{-}}+T_{C}\right)}{\left(P_{R} P_{C}\right)^{2}}\right]}{E\left[A^{\text {hop }}\right]},
$$

respectively. The end-to-end cost coefficients, $\mathcal{C}^{T}$ and $\mathcal{C}^{E}$, enable quantitative analysis on the cost-efficiency of an anycasting design. In the following sections, we use $\mathcal{C}^{T}$ and $\mathcal{C}^{E}$ as the main metrics to capture the end-to-end latency and energy consumption of anycasting designs using different prioritization and wake-up policies. 


\section{Cost-EFficient Prioritization Policy}

In this section, we analyze the impact of prioritization policies on the end-to-end cost-efficiency based on the endto-end cost model presented in Section IV. By separating the prioritization policy-independent and dependent components in the end-to-end cost coefficient expression, two greedy forwarding metrics are proposed for fully distributed forwarding decision targeting transmission latency and energy cost minimization, respectively.

\section{A. Determining Cost-Efficient Forwarding Metrics}

To evaluate how prioritization policy affects the end-toend cost coefficient, we first deduce the prioritization policyindependent components from (14) and (15). For (14), both $T^{c t r l}$ and $T^{d a t a}$ are fixed values for all sensor nodes. Therefore, they are independent of the prioritization policy in each hop. $P_{R}$ is the probability of the RTS packet being successfully received by at least one potential receiver. According to (2) and (4), $P_{R}$ is given as

$P_{R}=\sum_{n=1}^{\rho^{\prime}}\left(\begin{array}{l}\rho^{\prime} \\ n\end{array}\right)\left[1-\left(1-P_{R 0}\right)^{n}\right] P_{\text {wake }}^{n}\left(1-P_{\text {wake }}\right)^{\rho^{\prime}-n}$,

where $\rho^{\prime}=\frac{\rho}{2}$ is the number of neighboring nodes located within the forwarding region. Therefore, $P_{R}$ is determined by $P_{w a k e}$ and $\rho$, and thus is independent of the prioritization policy. We use $I_{1}^{T}$ and $I_{2}^{T}$ to represent the prioritization policyindependent components as $I_{1}^{T}=T^{c t r l} / P_{R}, I_{2}^{T}=T^{\text {data }}$, and thus the prioritization policy dependent components of $\mathcal{C}^{T}$ can be determined as $P_{C}, P_{D}, P_{A}$ and $E\left[A^{h o p}\right]$.

$$
\mathcal{C}^{T}=\frac{1}{E\left[A^{h o p}\right]} \cdot\left(\frac{I_{1}^{T}}{P_{C}}+\frac{I_{2}^{T}}{P_{D} \cdot P_{A}}\right) .
$$

To minimize $\mathcal{C}^{\mathcal{T}}$, a series of forwarding decisions need to be made end-to-end based on the prioritization policy to achieve global optimization. However, because of the distributed nature of anycasting operation, the forwarding decision can only be made at each hop with local information. Therefore, in order to minimize the end-to-end latency coefficient, $\mathcal{C}^{T}$, through distributed anycasting operation, we define

$$
\mathcal{F}_{i}^{T}=\frac{1}{\frac{I_{1}^{T}}{P_{C, i} \cdot A_{i}}+\frac{I_{2}^{T}}{P_{D, i} \cdot P_{A, i} \cdot A_{i}}}
$$

as the local forwarding metric used to prioritize the potential receivers, where $P_{C, i}, P_{D, i}, P_{A, i}$, and $A_{i}$ are the expected packet reception rates, and forwarding advancement achieved at hop $i$. These parameters can be decided locally if a particular sender and potential receiver pair is given [7], [15]. Upon receiving the RTS packet, each potential receiver evaluates its priority as the winning receiver using (18). The node with the largest $\mathcal{F}_{i}^{T}$ value gets the highest priority to become the winning receiver. The difference between (14) and (18) lies in the scope of optimization [15]. Since anycasting makes the forwarding decision in a fully distributed manner, (18) can been seen as a greedy forwarding metric that targets the endto-end latency coefficient minimization.

Similarly, for (15), if we represent the prioritization policyindependent components using $I_{1}^{E}=\frac{\varepsilon \cdot T^{c t r l}}{P_{R}}, I_{2}^{E}=2 \varepsilon \cdot T^{\text {data }}$,
TABLE I

AnALytical AND Simulation Evaluation SETtings

\begin{tabular}{|c|c|}
\hline \multicolumn{2}{|c|}{ Channel Model Parameters } \\
\hline Radio bandwidth & $250 \mathrm{kbps}$ \\
\hline Path loss exponent $\eta$ & 3 \\
\hline Shadow fading variance $\sigma$ & 4.5 \\
\hline Transmission power $P_{t}$ & $0 \mathrm{dBm}$ \\
\hline Noise power $P_{n}$ & $-100 \mathrm{dBm}$ \\
\hline Reference distance $D_{0}$ & $0.3 \mathrm{~m}$ \\
\hline Network Parameters \\
\hline RTS/CTS/ACK Packet Length & 10 byte \\
\hline DATA Packet Length & 50 byte \\
\hline Node Degree $\rho$ & 40 \\
\hline Duty-cycle $d_{c}$ & 0.1 \\
\hline
\end{tabular}

and $I_{3}^{E}=\frac{\varepsilon \cdot\left[\frac{1}{2} \rho_{I} T_{P \mid R}+P_{R}\left(\rho_{I}^{R} T_{R_{-} C}+T_{C}\right)\right]}{P_{R}^{2}}$, we can then determine prioritization policy dependent components of $\mathcal{C}_{i}^{E}$, as $P_{C}, P_{D}$, $P_{A}$ and $E\left[A^{h o p}\right]$, where

$$
\mathcal{C}_{i}^{E}=\frac{1}{E\left[A^{h o p}\right]} \cdot\left(\frac{I_{1}^{E}}{P_{C}}+\frac{I_{2}^{E}}{P_{D} P_{A}}+\frac{I_{3}^{E}}{P_{C}^{2}}\right) .
$$

Accordingly, a greedy forwarding metric, $\mathcal{F}_{i}^{E}$, is defined as

$$
\mathcal{F}_{i}^{E}=\frac{1}{\frac{I_{1}^{E}}{P_{C, i} \cdot A_{i}}+\frac{I_{2}^{E}}{P_{D, i} P_{A, i} \cdot A_{i}}+\frac{I_{3}^{E}}{P_{C, i}^{2} \cdot A_{i}}} .
$$

\section{B. Impact of varying forwarding metrics}

In this subsection, we demonstrate how different forwarding metrics prioritize the potential receivers differently within the disconnection distance of an anycasting sender based on log-normal channel model. The network and channel model parameters used in deriving the analytical results are listed in Table I to characterize the performance of MicaZ nodes [14] in an indoor environment. Unless otherwise noted, the parameters in Table I are used for deriving the numerical and simulation results in the rest of the paper.

Consider a single-hop anycasting operation initiated at sender $m$, as shown in Fig. 2. Upon receiving the preamble sent by $m$, located at $(0,0)$, any potential receiver within the disconnection distance evaluates its priority of becoming the winning receiver, using $\mathcal{F}$. If a potential receiver $i$, located at $(x, y)$, is selected as the next-hop (WR), the forwarding advancement $A_{i}$ achieved by this single-hop forwarding is given by $A_{i}(x, y)=x$. Therefore, based on the channel model in (1), the priority level of potential receiver $i$ can be derived using (18) and (20) as

$$
\begin{aligned}
\mathcal{F}^{T}(x, y) & =\frac{x}{\frac{I_{1}^{T}}{\bar{\Psi}\left(D, l_{C}\right)}+\frac{I_{2}^{T}}{\bar{\Psi}\left(D, l_{D}\right) \cdot \bar{\Psi}\left(D, l_{A}\right)}}, \\
\mathcal{F}^{E}(x, y) & =\frac{x}{\frac{I_{1}^{E}}{\bar{\Psi}\left(D, l_{C}\right)}+\frac{I_{2}^{E}}{\bar{\Psi}\left(D, l_{D}\right) \cdot \bar{\Psi}\left(D, l_{A}\right)}+\frac{I_{3}^{E}}{\bar{\Psi}\left(D, l_{C}\right)^{2}}},
\end{aligned}
$$

where $D=\sqrt{x^{2}+y^{2}} ; l_{C}, l_{D}, l_{A}$ are the CTS, DATA and ACK packet length. Using 21 , the expected $\mathcal{F}$ distribution within forwarding region can be derived. Fig. 3 shows the contour plot of $\mathcal{F}$ distribution in the upper-half of the forwarding region, where $x \geq 0$ and $y \geq 0$, of a sender located at $(0,0)$. The situation in the lower-half of the forwarding region, where $x \geq 0$ and $y \leq 0$, is symmetric to the situation 


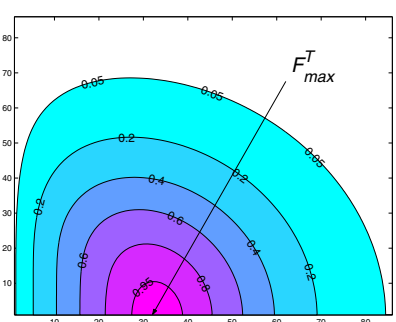

(a) $\mathcal{F}^{T}$

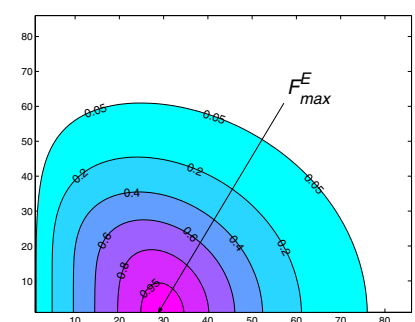

(b) $\mathcal{F}^{E}$

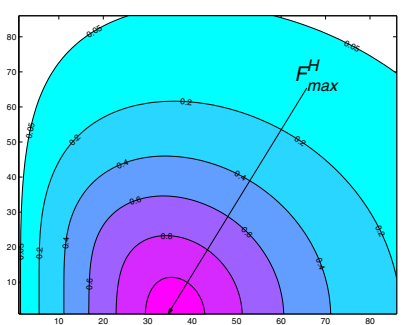

(c) $\mathcal{F}^{H}$

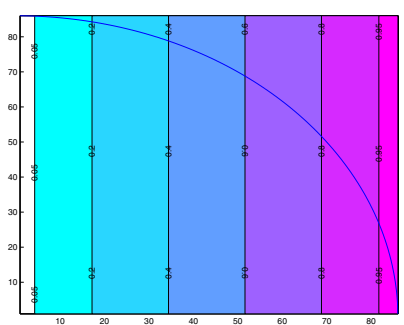

(d) $\mathcal{F}^{G}$

Fig. 3. Contour plots of the priority level distribution (normalized) using different forwarding metrics.

shown in Fig. 3. The expected $\mathcal{F}$ value has been normalized in the contour plot so that $0 \leq \mathcal{F} \leq 1$. The contour plots of the proposed forwarding metrics, $\overline{\mathcal{F}}^{T}$ and $\mathcal{F}^{E}$, are shown in Figs. 3 (a) and (b) separately based on our analytical model. The contour plots of a geographic forwarding metric proposed in [2] [6], $\mathcal{F}^{G}(x, y)=x$, and a heuristic forwarding metric proposed in [7], $\mathcal{F}^{H}(x, y)=\bar{\Psi}\left(x, l_{D}\right) * x$, are also shown in Figs. 3 (c) and (d), respectively, for comparison.

From Fig. 3, we observe that all channel-quality-aware forwarding metrics, $\mathcal{F}^{H}, \mathcal{F}^{T}$ and $\mathcal{F}^{E}$, consider the perhop cost and forwarding advance (cost-advancement) tradeoff while deciding the winning receiver from the forwarding set. Instead of prioritizing the potential receivers with larger forwarding advancement but lossy wireless link, the channelquality-aware forwarding metrics give higher priority to the the potential receivers located within the transient region of wireless channel [12]. According to the simulation results given in Fig. 6, such prioritization mechanisms help decrease about $60 \% \sim 90 \%$ the overall end-to-end communication cost compared with $\mathcal{F}^{G}$. From Fig. 3, we also observe that the achieved forwarding metric values can be maximized by a potential receiver located at a point $\left(0, y_{o}\right)$, pointed by the arrow in Fig. 3, for different channel-quality-aware forwarding metrics. The value of $y_{o}$ varies with different forwarding metrics and it is in inverse ratio to the gradient of the distribution contour. This implies that different cost minimization objectives, for example, latency or energy consumption, will lead to different forwarding decisions in considering the cost-advancement tradeoff. Generally, if the per-hop communication cost increases more dramatically with the increasing forwarding advancement, the resulting $y_{o}$ will decrease with a larger gradient of the distribution contour. The forwarding advancement achieved in single hop forwarding needs to be normalized by a particular cost model so that the greedy forwarding metric can be specified for particular endto-end cost minimization.

\section{COST-EFFicient WaKe-Up POLICY}

According to the end-to-end cost analysis, the preamble length $t_{P}$ determines the wake-up policy in an anycasting design if the duty-cycle operations are fixed for each sensor node in the network. In order to determine whether waiting for the most suitable neighboring node to wake up always results in overall cost-efficiency, the relationship between the preamble length and the achievable end-to-end cost coefficient needs to be understood. In this section, we first derive the cumulative distribution function $(\mathrm{CDF})$ of end-to-end cost coefficient, $\mathcal{C}$, using the cost-efficient forwarding metrics proposed in Section IV. Based on that we show how the expected end-to-end cost coefficient under certain confidence-levels can be controlled by preamble length adaptation under different network conditions.

\section{A. Distribution of End-to-End Energy and Latency Efficiency with Varying Preamble Length}

According to the basic anycasting operation specified in Section III.B, if $\mathcal{F}^{T}$ is the forwarding metric used in the forwarding decision, $E\left[\mathcal{F}^{T}\right]$ is the expected $\mathcal{F}_{i}^{T}$ value achieved by the winning receiver in single-hop anycasting. According to (12) and (13),

$$
E\left[\mathcal{F}_{i}^{T}\right]=1 / \mathcal{C}^{T}=D^{e 2 e} / E\left[C^{e 2 e}\right] .
$$

Since the $\mathcal{F}_{i}^{T}$ distribution within the disconnection distance of the sender is known according to (21), as shown in Fig. 3, the achieved $E\left[\mathcal{F}^{T}\right]$ in a single-hop forwarding must be less than a value $a$, if no potential receiver exists in the region enclosed by the $F^{T}$ contour of level $a$. Therefore, the probability of $E\left[\mathcal{F}^{T}\right]$ being less than a value $a$ is given as

$$
P\left(E\left[\mathcal{F}^{T}\right] \leq a\right)= \begin{cases}\left(1-\frac{A_{a}}{A}\right)^{\rho_{I}^{R}} & 0 \leq a \leq E\left[\mathcal{F}^{T}\right]_{\text {max }} \\ 0 & a<0 \\ 1 & a>E\left[\mathcal{F}^{T}\right]_{\text {max }}\end{cases}
$$

where $A$ is the area of region enclosed by the disconnection distance. $E\left[\mathcal{F}_{i}^{T}\right]_{\text {max }}$ is the maximum achievable $E\left[\mathcal{F}^{T}\right]$ value for a potential receiver located within $A . \rho_{I}^{R}$ is the expected number of nodes successfully receiving the RTS packet, i.e. the size of forwarding set, which is given in (5). $A(a)$ is the area of region enclosed by the $\mathcal{F}_{i}^{T}$ contour at level $a$, which is represented as an implicit function of $x$ and $y$. The area of the region enclosed by the contour at level $a$ can then be found using numerical analysis on (21).

Based on (22), the CDF of end-to-end latency coefficient, $\mathcal{C}^{T}$, achieved by an anycasting design using $F^{T}$ as the forwarding metric, is found as

$$
P\left(\mathcal{C}^{T}<c_{t}\right)=1-P\left(E\left[\mathcal{F}^{T}\right] \leq \frac{1}{c_{t}}\right) .
$$

Similarly, we derive the CDF of end-to-end energy coefficient, $\mathcal{C}^{E}$, achieved by an anycasting design under the forwarding metric, $F^{E}$, as

$$
P\left(\mathcal{C}^{E}<c_{e}\right)=1-P\left(E\left[\mathcal{F}^{E}\right] \leq \frac{1}{c_{e}}\right) .
$$




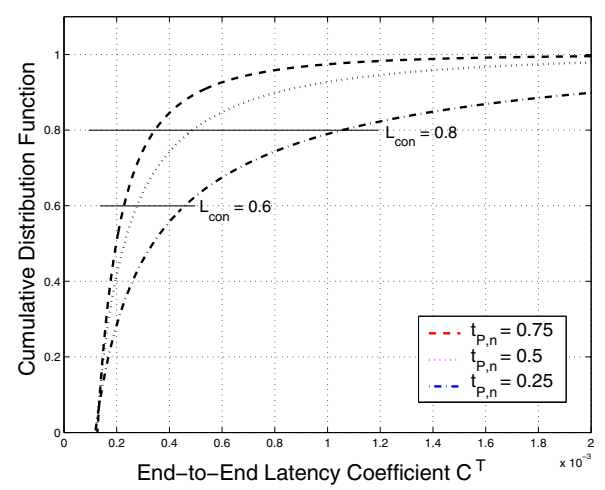

(a) $\mathrm{CDF}$ v.s. $\mathcal{C}^{T}$

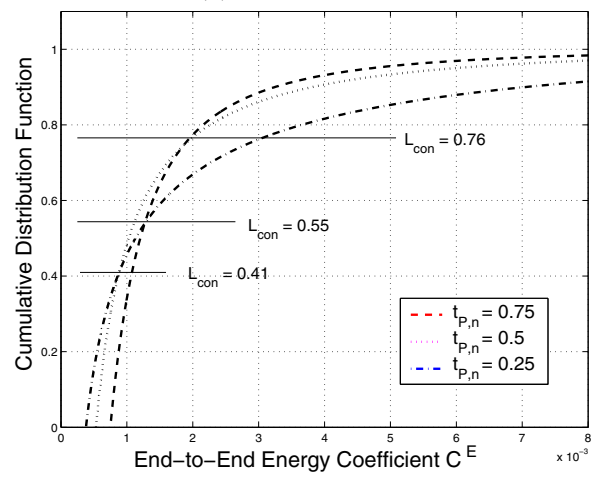

(b) $\operatorname{CDF}$ v.s. $\mathcal{C}^{E}$

Fig. 4. Cumulative distribution function of end-to-end latency coefficient $\mathcal{C}^{T}$ and end-to-end energy coefficient $\mathcal{C}^{E}$ with normalized preamble length equals to $0.25,0.5$ and 0.75 separately. $d_{c}=0.1$.

Equations (24) and (25) enable us to quantitatively analyze the end-to-end cost-efficiency of an anycasting design with specific design parameters in a statistical manner. According to the relationship between $E\left[C^{e 2 e}\right]$ and $\mathcal{C}$ shown in (22), a point on a CDF curve of $\mathcal{C}^{T}$ with confidence-level, $L_{\text {con }}=0.8$, and $\mathcal{C}^{T}=c_{t}$ indicates that $80 \%$ percent of the packets can be delivered with end-to-end latency less than $\left(c_{t} \cdot D^{e 2 e}\right)$. Similarly, a point on the CDF curve of $\mathcal{C}^{E}$ with confidencelevel, $L_{\text {con }}=0.6$, and $\mathcal{C}^{T}=c_{e}$ indicates that $60 \%$ percent of the packets can be delivered with end-to-end energy consumption less than $\left(c_{e} \cdot D^{e 2 e}\right)$. If we assume a fixed confidencelevel in the end-to-end cost analysis, for a set of CDF curves derived by using different anycasting design parameters, the one resulting in the minimum $\mathcal{C}$ value gives the anycasting design with the most cost-efficient design parameters.

\section{B. Determining Cost-Efficient Preamble Length}

In Fig. 4, the sample $\mathrm{CDF}$ plots of $\mathcal{C}^{T}$ and $\mathcal{C}^{E}$ are shown with varying normalized preamble lengths based on our analytical model. From Fig. 4, we find that the end-to-end communication cost can be controlled by varying the preamble length in anycasting design. Based on the analytical results shown in Fig. 4, we can make the following observations:

First, a larger normalized preamble length results in better end-to-end latency efficiency. According to Fig. 4 (a), by varying the $t_{P, n}$ from 0.75 to 0.25 , the achieved $\mathcal{C}^{T}$ is decreased from 1.4 to 0.35 at $L_{c o n}=0.8$, which leads to an about 4 times increase in end-to-end latency efficiency. The analytical

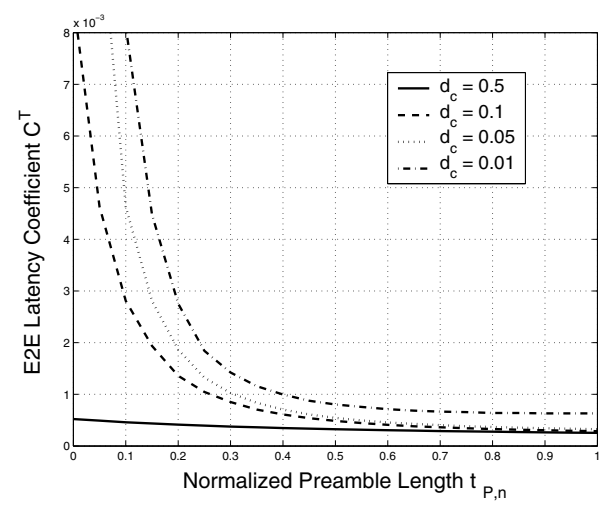

(a) $\mathcal{C}^{T}$ v.s. $t_{P, n},\left(L_{\text {con }}=0.9\right)$

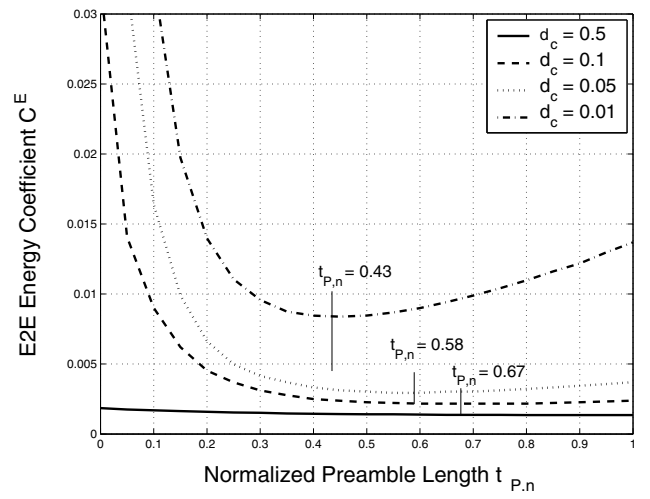

(b) $\mathcal{C}^{E}$ v.s. $t_{P, n},\left(L_{\text {con }}=0.9\right)$

Fig. 5. The relationship between the end-to-end cost coefficient $\mathcal{C}$ achieved at confidence-level $L_{c o n}=0.9$ and the corresponding preamble length $t_{P}$. The duty-cycle $d_{c}$ is varied from 0.01 to 0.5 .

results indicate that although increasing the preamble length introduces extra delay in waking up the neighboring node, the overall transmission delay can be decreased with smaller achievable $\mathcal{C}^{T}$ value due to a better winning receiver becoming available in a larger forwarding set.

Second, a larger normalized preamble length cannot guarantee better end-to-end energy efficiency. From Fig. 4 (b), we observe that, compared with end-to-end latency cost, the achieved end-to-end energy coefficient does not monotonously decrease with increasing preamble length, for example, at $L_{\text {con }}=0.6$. The analytical results indicate that a longer preamble length results in more neighboring nodes being awakened to receive the $R T S$ packet; thus, more energy is consumed at the anycasting receivers. On the other hand, a longer preamble length results in larger $\rho_{I}^{R}$ and leads to a higher probability of achieving a specific end-to-end energy coefficient. The tradeoff between waking up more neighboring nodes to achieve a better $\mathcal{C}^{E}$ value and consuming more energy in each transmission attempt leads to an optimal $t_{P, n}<1$ for minimizing the end-to-end energy coefficient.

To fully investigate the relationship between normalized preamble length $t_{P, n}$ and the achieved normalized end-toend cost coefficient $\mathcal{C}$ in a larger anycasting design parameter space, Fig. 5 is plotted based on our cost model to show the situation when an anycasting design operates in high $\left(d_{c}=0.5\right)$, low $\left(d_{c}=0.1\right.$ and $\left.d_{c}=0.05\right)$, and extremely 
low $\left(d_{c}=0.01\right)$ duty-cycled WSNs.

High duty-cycled WSNs $\left(d_{c} \simeq 1\right)$ : The end-to-end costefficiency is not sensitive to the preamble length adaptation. When the normalized preamble length is increased from 0 to 1 , both $\mathcal{C}^{T}$ and $\mathcal{C}^{E}$ are hardly changed. Therefore, in high dutycycled MicaZ network, the cost-efficiency of an anycasting design cannot be significantly improved through preamble length adaptation.

Low duty-cycled WSNs $\left(d_{c} \simeq 0.1\right)$ : The end-to-end costefficiency is sensitive to preamble length adaptation, especially when $t_{P, n}$ is relatively small. According to the analytical results in Fig. 5, when the normalized preamble length $t_{P, n}$ increases from 0 to 0.4 , both end-to-end latency and energy coefficient can be decreased for about $90 \%$. For the scenarios when $d_{c}=0.05$ and $d_{c}=0.01$, the end-to-end energy coefficient $\mathcal{C}^{E}$ achieved at a confidence level 0.9 can be minimized with preamble length $t_{P, n}=0.67$ and $t_{P, n}=0.58$ respectively. However, both $\mathcal{C}^{E}$ and $\mathcal{C}^{T}$ are relatively stable when $t_{P, n}$ changes in $[0.4,1]$. Accordingly, $t_{P} \geq 0.4$ is recommended for anycasting design to achieve high end-toend cost-efficiency in low duty-cycled MicaZ network.

Extremely low duty-cycled WSNs $\left(d_{c} \simeq 0.01\right)$ : The end-toend cost-efficiency is extremely sensitive to preamble length adaptation, especially when $t_{P, n}$ is relatively small. According to the analytical results shown in Fig. 5, $\mathcal{C}^{E}$ is minimized with a smaller $t_{P, n}$, before $\mathcal{C}^{T}$ is minimized while $t_{P, n}$ is increased from 0 to 1 . If we denote the $t_{P, n}$ value for $\mathcal{C}^{E}$ minimization as $t^{o}$, both $\mathcal{C}^{E}$ and $\mathcal{C}^{T}$ can be decreased dramatically while $t_{P, n}$ increases within $\left[0, t^{o}\right]$. However, for the cases when $t_{P, n}$ increases within $\left[t^{o}, 1\right], \mathcal{C}^{E}$ increases dramatically with slightly decreased $\mathcal{C}^{T}$. Such analytical results indicate that the communication cost introduced by waking up neighboring nodes contributes more in the overall energy consumption for end-to-end packet delivery in lower duty-cycled WSNs. Therefore, waiting for the most suitable neighboring node to wake up does not guarantee overall energy efficiency. Based on the above analysis, $t_{P}=t^{o}$ is recommended for anycasting design to achieve high end-to-end cost-efficiency in extremely low duty-cycled network. According to our analytical model, when $d_{c}=0.01, t^{\circ}=0.43$.

\section{Simulation VALidation}

In this section, we present the simulation results obtained in a wireless network simulator (GlomoSim) with realistic channel model to validate the cost-efficiency of our proposed forwarding metrics and preamble length control guidelines derived based on our cost model. The physical and link layers are designed to follow the log-normal channel model. The anycasting implementation follows the basic anycasting operation described in Section II.A. The simulation parameters remain the same as shown in Table I.

\section{A. Validation of Cost-Efficient Prioritization Policy}

In this simulation senario, the end-to-end communication cost $E\left[C^{e 2 e}\right]$, in terms of transmission latency and network energy consumption, are collected for 5000 packet deliveries from random anycasting senders to the sink using forwarding

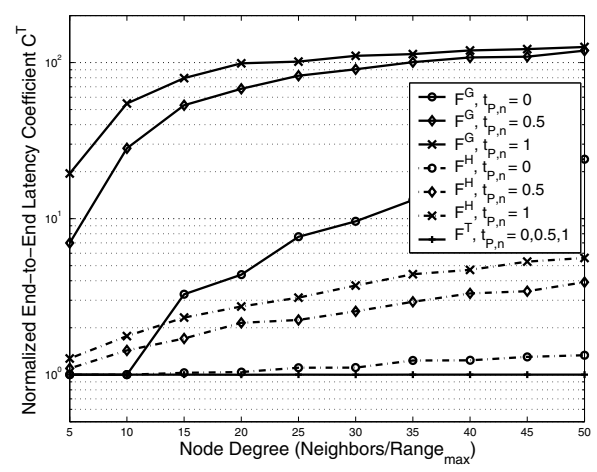

(a) Normalized Latency Coefficient v.s. $\rho$

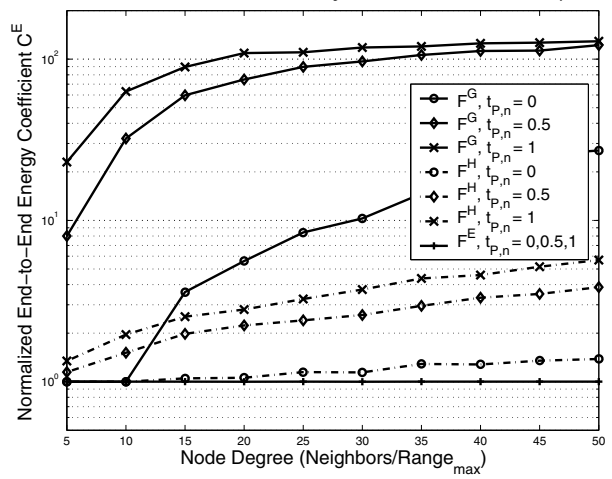

(b) Normalized Energy Coefficient v.s. $\rho$

Fig. 6. Comparing the normalized end-to-end latency coefficient $\mathcal{C}^{T}$ and the energy coefficient $\mathcal{C}^{E}$ achieved by different forwarding metrics $\mathcal{F}^{T}, \mathcal{F}^{E}, \mathcal{F}^{H}$ and $\mathcal{F}^{G}$ with varying network node density.

metric $\mathcal{F}^{T}, \mathcal{F}^{E}, \mathcal{F}^{G}$ and $\mathcal{F}^{H}$. The maximum end-to-end transmission distance is $200 \mathrm{~m}$. According to (12), the average $\left(E\left[C^{e 2 e}\right] / D^{e 2 e}\right)$ values obtained in 5000 packet deliveries are calculated to show the end-to-end cost coefficient achieved by using different forwarding metrics in anycasting operation. Since we focus only on the cost-efficiency variation due to changing forwarding metrics in an anycasting design, we want to keep the prioritization policy-independent parameters of anycasting design to be constant. According to (17) and (19), $P_{R}, \rho_{I}$ and $\rho_{I}^{R}$ are the prioritization policy-independent components affecting $\mathcal{C}$, and the preamble length $t_{P}$ is the only anycasting design parameter that affects these values. Therefore, a constant preamble length is used to validate the cost-efficiency of different forwarding metrics. Three simulation scenarios with $t_{P, n}=0, t_{P, n}=0.5$ and $t_{P, n}=1$ are constructed respectively to span the entire design space of the preamble length.

Fig. 6 (a) shows the relative end-to-end latency efficiency of $\mathcal{F}^{G}$ and $\mathcal{F}^{H}$ with respect to $\mathcal{F}^{T}$ metric, i.e., the $\mathcal{C}^{T}$ values achieved by metrics $\mathcal{F}^{G}$ and $\mathcal{F}^{H}$ are normalized to the latency cost coefficient achieved by $\mathcal{F}^{T}$. Fig. 6(b) shows the relative end-to-end energy efficiency of $\mathcal{F}^{G}$ and $\mathcal{F}^{H}$ with respect to $\mathcal{F}^{E}$ metric. The simulation results validate that, compared with geographic forwarding metric $\mathcal{F}^{G}$, the proposed forwarding metrics, $\mathcal{F}^{T}$ and $\mathcal{F}^{E}$, can reduce more than $90 \%$ the end-to-end latency and energy consumption, respectively. Compared with heuristic forwarding metric, $\mathcal{F}^{H}$, 
TABLE II

AVERAGE END-TO-END LATENCY COEFFICIENT $(m s / M)$ AND ENERGY COEFFICIENT $(m J / M)$

\begin{tabular}{|c|c|c|c|c|c|c|}
\hline & \multicolumn{2}{|c|}{$d_{c}=0.01$} & \multicolumn{2}{c|}{$d_{c}=0.1$} & \multicolumn{2}{c|}{$d_{c}=0.5$} \\
\hline & $\mathcal{C}^{T}$ & $\mathcal{C}^{E}$ & $\mathcal{C}^{T}$ & $\mathcal{C}^{E}$ & $\mathcal{C}^{T}$ & $\mathcal{C}^{E}$ \\
\hline$t_{P, n}=0.8$ & 0.36 & 56 & 0.16 & 20.4 & 0.17 & 9.1 \\
\hline$t_{P, n}=0.4$ & 0.41 & 42.5 & 0.24 & 16.1 & 0.17 & 8.5 \\
\hline$t_{P, n}=0.1$ & 1.82 & 87.7 & 0.8 & 37.7 & 0.19 & 10 \\
\hline
\end{tabular}

$\mathcal{F}^{T}$ and $\mathcal{F}^{E}$ can reduce the end-to-end latency and energy consumption for anycasting with long preamble length by about $25 \%$, with moderate preamble length by about $55 \%$, and with short preamble length by about $65 \%$, in the network with moderate node degree. From the simulation results, we can also observe that the cost-coefficient reduction achieved by using the proposed forwarding metrics increase with larger node density or preamble length, i.e., the cost-efficiency is more sensitive to the forwarding metric when the node density is high or the preamble length is large. The reason for this behavior is that the expected number of potential receivers decreases with smaller node density and preamble length. Accordingly, different forwarding metrics tend to result in the same winning receiver even when the prioritization policy is different. When the expected number of potential receivers approaches 1 , the end-to-end cost-efficiency will not be affected by the forwarding metric adopted by an anycasting design.

\section{B. Validation of Cost-Efficient Wake-Up Policy}

The cost-efficient preamble length derived based on our analytical work is validated through simulations by comparing the end-to-end performance of anycasting designs with preamble length $t_{P, n}=0.1, t_{P, n}=0.4$ and $t_{P, n}=0.8$. Three simulation scenarios are constructed with $d_{c}=0.01, d_{c}=0.1$ and $d_{c}=0.5$ respectively, to capture the case of high, low and extremely low duty-cycled WSN environment. The endto-end communication cost $E\left[C^{e 2 e}\right]$, in terms of transmission latency and network energy consumption, are collected for 5000 packet deliveries from random anycasting senders to the sink with $D^{e 2 e} \leq 200 \mathrm{~m}$. We used the average $\left(E\left[C^{e 2 e}\right] / D^{e 2 e}\right)$ values obtained in 5000 packet deliveries to show the endto-end cost-efficiency achieved by anycasting designs with different normalized preamble lengths under different dutycycle operations. The simulation results shown in Table II confirm that,

- For high-duty cycled WSNs, varying the preamble length does not help improve the end-to-end performance of anycasting design significantly.

- For low-duty cycled WSNs, compared with the case when only short RTS packets in sequence are used to wake up the neighboring node, using a normalized preamble length of $t_{P, n} \geq 0.4$ can improve the performance of anycasting design by reducing about $70 \%$ the end-to-end latency and $57 \%$ the energy costs.

- For extremely low-duty cycled WSNs, compared with the case when a long preamble or only short RTS packets in sequence are used to wake up the neighboring node, using a normalized preamble at length of around $t_{P, n}=0.4$ can significantly improve the end-to-end performance of anycasting design by reducing about $51 \%$ of the end-toend energy and $76 \%$ the latency costs.

\section{CONCLUSION}

In this paper, we present an end-to-end analysis framework to quantitatively assess the latency and energy efficiency of anycasting operation under log-normal channel model. Based on the proposed analytical model, two greedy forwarding metrics, $\mathcal{F}^{T}$ and $\mathcal{F}^{E}$, are proposed to target end-to-end latency and energy consumption efficiency in a fully distributed receiver contention process. According to our performance analysis, the proposed forwarding metrics help reduce the endto-end latency and energy consumption by about $55 \%$ for anycasting with moderate preamble length, compared with the existing heuristic forwarding metrics in [7]. Using the proposed forwarding metrics, we further investigate the anycasting wake-up policy design problem. A series of preamble length control guidelines are proposed based on our analytical model to reduce, by more than half, the end-to-end energy and latency cost through tuning the anycasting preamble length in low and extremely-low duty-cycled WSNs.

\section{REFERENCES}

[1] J. Kim, X. Lin, N. B. Shroff et al., "Optimal anycast technique for delaysensitive energy-constrained asynchronous sensor networks," in Proc. of IEEE Infocom'09, April 2009.

[2] M. Zorzi and R. Rao, "Geographic random forwarding (GeRaf) for ad hoc and sensor networks: multihop performance," IEEE Transactions on Mobile Computing, no. 4, December 2003.

[3] — "Geographic random forwarding (GeRaf) for ad hoc and sensor networks: multihop performance," IEEE Trans. on Mobile Computing, no. 4, December 2003.

[4] R. C. Shah, S. Wietholter, A. Wolisz et al., "Modeling and analysis of opportunistic routing in low traffic scenarios," in Proc. of WIOPT '05, Washington, DC, April 2005, pp. 294-304.

[5] J. Kim, X. Lin, N. B. Shroff, and P. Sinha, "On maximizing the lifetime of delay-sensitive wireless sensor networks with anycast," in Proc. of IEEE Infocom'08, Phoenix, Arizona, April 2008.

[6] S. Liu, K.-W. Fan, and P. Sinha, "CMAC: Energy efficient MAC layer design for sensor networks with anycasting," to appear in IEEE Transactions on Sensor Networks, March 2009.

[7] M. Zuniga, S. Karim, K. Bhaskar et al., "Efficient geographic routing over lossy links in wireless sensor networks," ACM Trans. Sen. Netw., vol. 4, no. 3, pp. 1-33, May 2008.

[8] J. Polastre, J. Hill, and D. Culler, "Versatile low power media access for wireless sensor networks," in Proc. of SenSys '04, New York, NY, November 2004, pp. 95-107.

[9] Y. Xue, B. Ramamurthy, and M. C. Vuran, "A service-differentiated realtime communication scheme for wireless sensor networks," in Proc. of SenseApp '08, Montreal, Canada, October 2008.

[10] S. Biswas and R. Morris, "ExOR: opportunistic multi-hop routing for wireless networks," in Proc. of SIGCOMM '05, New York, NY, January 2005, pp. 133-144.

[11] R. Zheng and C. Li, "How good is opportunistic routing?: a reality check under Rayleigh fading channels," in Proc. of MSWiM '08, New York, NY, October 2008, pp. 260-267.

[12] M. Zuniga and B. Krishnamachari, "An analysis of unreliability and asymmetry in low-power wireless links," ACM Trans. Sen. Netw., vol. 3, no. 2, p. 7, June 2007.

[13] Chipcon, "Datasheet for chipcon (TI)CC2420 2.4 ghz IEEE 802.15.4/zigbee RF transceiver," 2006. [Online]. Available: http://ti.com

[14] "MicaZ wireless module." [Online]. Available: http://www.xbow.com/ Products/Product_pdf_files/Wireless_pdf/MICAz_Datasheet.pdf

[15] S. Lee, B. Bhattacharjee, and S. Banerjee, "Efficient geographic routing in multihop wireless networks," in Proc. of MobiHoc '05. New York, NY, USA: ACM, 2005, pp. 230-241. 